asthma, as the numbers of $i \mathrm{NKT}$ cells detected in the bronchoalveolarlavage fluid from patients with sarcoidosis - a multisystem disorder that mainly affects the lungs - were similar to the numbers found in healthy individuals.

The $i$ NKT cells from the lungs of patients with asthma produced the $\mathrm{T}_{\mathrm{H}} 2$ cytokines interleukin-4 (IL-4) and IL-13 but produced very small amounts of interferon- $\gamma$. By contrast, $i$ NKT cells from healthy individuals or patients with sarcoidosis produced all three cytokines.

Therefore, a subgroup of $i \mathrm{NKT}$ cells - which express CD4 and produce $\mathrm{T}_{\mathrm{H}} 2$ cytokines - are recruited to or are clonally expanded in the lungs of patients with asthma and produce cytokines that are essential to the development of this disorder.

Olive Leavy

ORIGINAL RESEARCH PAPER Akbari, O. et al. $\mathrm{CD}^{+}$invariant T-cell-receptor ${ }^{+}$natural killer T cells in bronchial asthma. N. Engl. J. Med. 354, 1117-1129 (2006)

viruses (including influenza virus) and a positive-sense ssRNA virus, Japanese encephalitis virus (which is a flavivirus). By contrast, MDA5, but not RIG-I, was required for the detection of a positive-sense ssRNA virus, encephalomyocarditis virus (which is a picornavirus). Moreover, RIG-I-deficient mice and MDA5deficient mice were highly susceptible to infection with the respective viruses, confirming that this receptor-mediated viral recognition has an important role in host defence.

MDA5 and RIG-I are therefore crucial for the recognition of different groups of viruses. How these receptors detect different viral RNAs remains unclear, and the authors suggest that analysing the crystal structures of the helicase (RNA-binding) domains of these proteins might shed light on the molecular mechanisms of this differential recognition.

Davina Dadley-Moore

ORIGINAL RESEARCH PAPER Kato, H. et al. Differential roles of MDA5 and RIG-I helicases in the recognition of RNA viruses. Nature 9 Apr 2006 (doi:10.1038/nature04734)

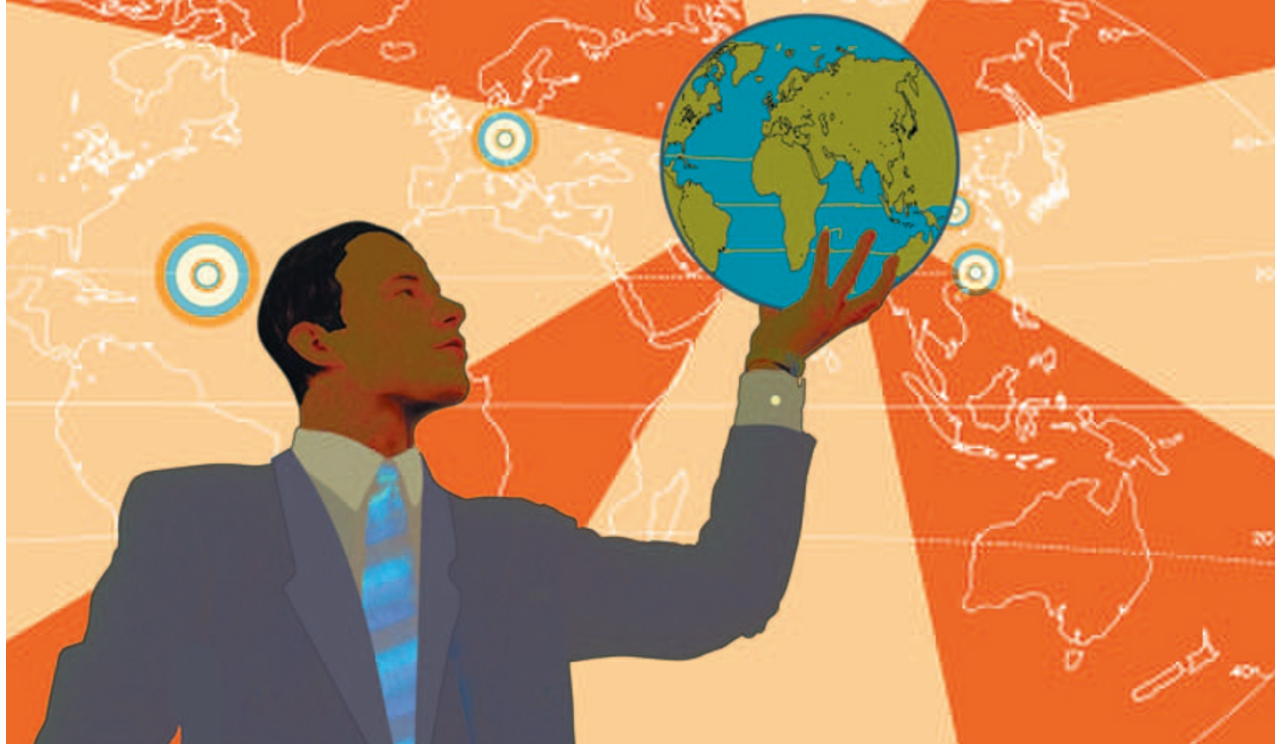

$\Rightarrow$ T CELLS

\title{
BLIMP1 increases its control over lymphocytes
}

The transcriptional repressor B-lymphocyteinduced maturation protein 1 (BLIMP1) is the 'master regulator' of the terminal differentiation of B cells into plasma cells. However, two papers published recently in Nature Immunology now report that BLIMP1 also has a key role in regulating $\mathrm{T}$-cell homeostasis and activation.

Although the function of BLIMP1 in B cells has been well characterized, the function of BLIMP1 in other cell types has not been studied in detail. To address this gap in our knowledge, Kallies et al. generated BLIMP1-reporter mice, in which expression of a bicistronic DNA construct expressing the genes encoding green fluorescent protein (GFP) and a non-functional form of BLIMP1 was 'knocked in' to the gene encoding BLIMP1 (Prdm1). Mice homozygous for this construct lack BLIMP1 function and die in utero; therefore, subsequent analysis in this study was carried out using recombination-activating gene 1 (RAG1)-deficient mice reconstituted with fetal-liver cells from the knock-in mice (denoted Prdm19fp/gfp mice). By contrast, Martins et al. generated mice lacking BLIMP1 expression only in cells of the T-cell lineage (denoted CKO mice).

Both groups observed lymphocytic infiltration of the colon, lungs and liver, and tissue destruction indicative of colitis in the colon of their mice. Consistent with this inflammatory phenotype, $\mathrm{CD}^{+} \mathrm{T}$ cells from these mice produced more interferon- $\gamma$ than control cells when stimulated in vitro and the total number of effector $\mathrm{CD}^{+} \mathrm{T}$ cells was increased in the spleen and lymph nodes of both Prdm $1^{\text {gfp/gfp }}$ and CKO mice. Furthermore, analysis of GFP expression in CD4 ${ }^{+} \mathrm{T}$ cells from the Prdm1 19f/gfp BLIMP1-reporter mice and Prdm1 mRNA in CD4 ${ }^{+} \mathrm{T}$ cells from wildtype mice indicated that among $\mathrm{CD} 4^{+} \mathrm{T}$ cells, effector cells expressed the highest levels of BLIMP1. Evidence that the phenotypes of colitis and an expanded effector $\mathrm{CD} 4^{+} \mathrm{T}$-cell population were a result of BLIMP1 having an intrinsic T-cell function (that is, an intrinsic role in regulating T-cell homeostasis) was provided by several observations. First, naive CD4 ${ }^{+} \mathrm{T}$ cells from CKO mice, but not Prdm $1^{\text {gfp/gfp }}$ mice, hyperproliferated when stimulated in vitro under sub-optimal conditions. Second, effector $\mathrm{CD} 4^{+}$and $\mathrm{CD} 8^{+}$ T cells from Prdm $1^{\text {gfp/gfp }}$ mice hyperproliferated when stimulated with CD3- and CD28-specific antibody in the presence of cytokines. Last, co-transfer of wild-type and Prdm $1^{\text {gfp/gfp }} \mathrm{CD} 4^{+}$ $T$ cells to RAG1-deficient mice resulted in the preferential expansion of the $\mathrm{CD} 4{ }^{+} \mathrm{T}$-cell population lacking functional BLIMP1.

Colitis and expanded effector CD4 ${ }^{+} \mathrm{T}$-cell populations are also characteristics of mice lacking $\mathrm{CD} 4{ }^{+} \mathrm{CD} 25^{+}$regulatory $\mathrm{T}\left(\mathrm{T}_{\mathrm{Reg}}\right)$ cells. However, both Prdm $1^{\text {gfp/gfp }}$ and CKO mice had $\mathrm{T}_{\text {Reg }}$-cell populations, and these cells were functional in in vitro assays of regulatory function. By contrast, although $\mathrm{T}_{\text {Reg }}$ cells from Prdm1 1fp/gfp mice showed normal regulatory function in vivo, $\mathrm{T}_{\text {Reg }}$ cells from CKO mice had impaired regulatory function in vivo.

Although several points remain to be clarified (including defining the molecular mechanisms by which BLIMP1 mediates its effects in T cells, more precisely defining the $\mathrm{T}_{\text {Reg }}$-cell phenotype in the absence of BLIMP1 and determining whether BLIMP1 has a role in T-cell development in the thymus), these two studies define a previously unknown function for BLIMP1 as a key regulator of T-cell homeostasis and activation.

Karen Honey

ORIGINAL RESEARCH PAPERS Martins, G. A. et al.

Transcriptional repressor Blimp-1 regulates T cell homeostasis and function. Nature Immunol. 26 Mar 2006 (doi:10.1038/ni1320) | Kallies, A. et al. Transcriptional repressor Blimp-1 is essential for T cell homeostasis and self-tolerance. Nature Immunol. $26 \mathrm{Mar} 2006$ (doi:10.1038/ni1321) 